\title{
Hyperhomocysteinemia enhances vascular inflammation and accelerates atherosclerosis in a murine model
}

\author{
Marion A. Hofmann, ${ }^{1}$ Evanthia Lalla, ${ }^{1}$ Yan Lu, ${ }^{1}$ Michelle Ryu Gleason, ${ }^{1}$ \\ Bonnie M. Wolf, ${ }^{1}$ Nozomu Tanji, ${ }^{1}$ Luis J. Ferran, Jr., ${ }^{1}$ Brigitte Kohl, ${ }^{2}$ Vijay Rao, ${ }^{3}$ \\ Walter Kisiel, ${ }^{4}$ David M. Stern, ${ }^{1}$ and Ann Marie Schmidt ${ }^{1}$
}

${ }^{1}$ College of Physicians and Surgeons, Columbia University, New York, New York, USA

${ }^{2}$ Department of Internal Medicine, University of Heidelberg, Heidelberg, Germany

${ }^{3}$ University of Texas Health Sciences Center at Tyler, Tyler, Texas, USA

${ }^{4}$ University of New Mexico School of Medicine, Albuquerque, New Mexico, USA

Address correspondence to: Marion A. Hofmann, College of Physicians and Surgeons, Columbia University, 630 W. 168th Street, P\&S 17-501, New York, New York 10032, USA.

Phone: (212) 305-6406; Fax: (212) 305-5337; E-mail: mah81@columbia.edu.

Received for publication June 15, 2000, and accepted in revised form December 21, 2000.

\begin{abstract}
Although hyperhomocysteinemia (HHcy) is a well-known risk factor for the development of cardiovascular disease, the underlying molecular mechanisms are not fully elucidated. Here we show that induction of HHcy in apoE-null mice by a diet enriched in methionine but depleted in folate and vitamins B6 and B12 increased atherosclerotic lesion area and complexity, and enhanced expression of receptor for advanced glycation end products (RAGE), VCAM-1, tissue factor, and MMP-9 in the vasculature. These homocysteine-mediated (HC-mediated) effects were significantly suppressed, in parallel with decreased levels of plasma HC, upon dietary supplementation with folate and vitamins B6/B12. These findings implicate HHcy in atherosclerotic plaque progression and stability, and they suggest that dietary enrichment in vitamins essential for the metabolism of $\mathrm{HC}$ may impart protective effects in the vasculature.
\end{abstract}

J. Clin. Invest. 107:675-683 (2001).

\section{Introduction}

Multiple epidemiologic studies have indicated that elevated levels of plasma homocysteine $(\mathrm{HC})$ portend increased risk of cardiovascular disease and stroke (1-2), however, the molecular pathogenesis underlying these observations in vivo has yet to be fully defined. Studies from in vitro and in vivo investigation have suggested that generation of potent reactive oxygen intermediates, such as superoxide anion radical and hydrogen peroxide and impaired production of endothelial nitric oxide, are central mechanisms by which vascular exposure to elevated levels of HC may mediate long-term oxidative damage at the vascular interface (3-5). In vivo, moderate hyperhomocysteinemia (HHcy) induced by methionine loading in human subjects resulted in impaired endotheliumdependent vasodilatation in response to infusion of acetylcholine into the brachial artery, a process suppressed in the presence of the antioxidant ascorbic acid (6). Other studies have suggested that elevated levels of HC stimulate the proliferation of smooth muscle cells (7) and the enhanced oxidation of LDL (8). Furthermore, HHcy is associated with a prothrombotic state, as suggested by increased aggregation of platelets $(1,9)$, enhanced generation of tissue factor (TF) (9), increased activation of coagulation factors, such as factors V, X, and XII, along with decreased activation of protein $C$ and cell-surface thrombomodulin (10-12), and modulation of tissue plasminogen activator binding to its endothelial receptor, annexin II (13-14). These findings strongly suggested that HHcy likely contributes, in part, to enhanced vascular inflammation and hypercoagulability, factors intimately linked to the development of atherosclerosis and associated thrombotic events.

To test these concepts, we employed apoE-null mice in whom atherosclerosis develops on standard rodent chow (15-16). Mice were rendered hyperhomocysteinemic upon consumption of a diet enriched in methionine and significantly depleted in vitamins essential for the metabolism of $\mathrm{HC}$, folate, and vitamins B6/B12. In addition, to address a central, yet unanswered question in the clinic, whether dietary supplementation with vitamins essential for the metabolism of HC (17-18) suppresses HC-mediated accelerated vascular disease, we fed apoE-null mice a diet enriched in both methionine and vitamins folate, $\mathrm{B} 6$, and $\mathrm{B} 12$. We employed this model to determine the effect of HHcy on vascular inflammation and atherogenesis.

\section{Methods}

Induction of HHcy. Male apoE-null mice (15-16) (backcrossed ten generations into C57BL/6J) were obtained from The Jackson Laboratories (Bar Harbor, Maine, USA). At age 4 weeks, mice were fed standard rodent chow 5001C (diet A), a diet enriched in methionine 
with low levels of folate, vitamins B6, and B12 (diet B) (Harlan Teklad TD97345; Harlan Teklad, Madison, Wisconsin, USA), or a diet enriched in methionine and folate, vitamins B6 and B12 (diet C) (Harlan Teklad TD98002). To control for the possibility that dietary deficiency in folate, vitamins B6, and B12 alone might modulate atherogenesis, we fed an additional group of mice a diet with low levels of folate, vitamins B6, and B12 (diet D) (Harlan Teklad TD00428). All diets were matched for kilocalories, and mice were allowed free access to food and water. Mice were sacrificed after 8 weeks on the diet.

Assessment of atherosclerotic lesion area. Upon sacrifice, blood was removed from the inferior vena cava into EDTA (final concentration, $0.05 \mathrm{M}$ ), and plasma/red blood cells were retrieved. The aorta was perfused gently with PBS in a retrograde manner, and the heart was removed and stored in buffered formalin (10\%). Cryostat sections were prepared and embedded in gelatin (25\%). Serial sections, $10-\mu \mathrm{m}$ thick, were cut from the level of the aortic valve leaflets up to about $480 \mu \mathrm{m}$ above the leaflets in the aortic sinus, every other section was retrieved and placed onto gelatin-coated slides (5\%), and four sections were placed onto each slide for a total of five slides. Sections were stained with oil red $\mathrm{O}$ and hematoxylin/light green. Quantitation of atherosclerotic lesion area was performed on one section from each slide (beginning at the site where three distinct valves first appear) using a Zeiss microscope and Zeiss Image (Zeiss, Thornwood, New York, USA); mean lesion area from slides two through five is reported (19). Complex atherosclerotic lesions were defined as the presence of fibrous caps, cholesterol clefts, or lesion necrosis (19).

Assessment of elastolysis. van Giessen-stained cross-sections from the aortic sinus were prepared, and quantification for extent of elastolysis/aneurysm formation was performed (20-21). Ectasis of the media underlying intimal atherosclerotic plaques was graded as follows: stage 0 , no elastolysis despite intimal lesion; stage 1 , elastolysis of the internal elastic lamina; stage 2, elastolysis of the internal elastic lamina plus one or more elastic layers within the tunica media; stage 3, elastolysis of the internal elastic lamina, all elastic layers within the tunica media, as well as the external elastic lamina; and stage 4, elastolysis of all elastic layers and bulging into the adventitia.

Plasma and red blood cell analyses. Plasma was evaluated for levels of glucose, cholesterol, triglyceride, and creatinine by Analytics Inc. (Gaithersburg, Maryland, USA). Glycosylated hemoglobin (\%) was determined on red blood cell lysates (Pierce Chemical Co., Arlington Heights, Illinois, USA). Levels of $\mathrm{HC}$, folate, and vitamins B12/B6 were performed as described (22). Levels of plasma TNF- $\alpha$ were determined using an ELISA from R\&D Systems Inc. (Minneapolis, Minnesota, USA).

Electromobility shift assay. Nuclear extracts were prepared as described (23). A radiolabeled ${ }^{32} \mathrm{P}$ consensus probe for
NF- $\kappa \mathrm{B}$ was incubated with nuclear extracts; the resulting complexes were subjected to electrophoresis by PAGE (6\%), and autoradiography was performed.

Immunoblotting and zymography. At sacrifice, aorta (aortic sinus to the mid-thoracic region) and kidney were retrieved. Immunoblotting was performed by subjecting tissue lysate (24) to immunoblotting with anti-receptor for advanced glycation endproducts (anti-RAGE) IgG (24), anti-EN-RAGE IgG (24), anti-VCAM-1 IgG (Santa Cruz Biotechnology Inc., Santa Cruz, California, USA), anti-TF IgG (prepared by Walter Kisiel and Vijay Rao), and anti-MMP-9 IgG (Oncogene Research Products, Cambridge, Massachusetts, USA). Zymography was performed on aortic lysates using gelatin-laden gels (Invitrogen, Carlsbad, California, USA).

Immunohistochemistry. Aortic tissue was retrieved and fixed with formalin; paraffin-embedded sections $(5-\mu \mathrm{m}$ thick) were prepared and subjected to immunohistochemistry with anti-TF IgG, anti-Mac 3 IgG (Sigma Chemical Co., St. Louis, Missouri, USA), anti-VCAM-1 IgG, anti-RAGE IgG (24), and anti-EN-RAGE IgG (24). Experiments in which equal amounts of nonimmune IgG were included revealed no specific immunostaining (data not shown).

In vitro analyses. Human umbilical vein endothelial cells (HUVECs) were prepared and characterized as described (24). Cells in the presence of FBS $(0.5 \%)$ were exposed to BSA, L-HC, or L-cysteine (Sigma Chemical Co.) as indicated. In certain experiments, electrophoretic mobility shift assays as above were performed; in others, immunoblotting was performed for detection of RAGE and TF antigens.

Statistical analysis. All data are reported as mean plus or minus SD. Where indicated, densitometric analysis of band intensity was performed using ImageQuant (Molecular Dynamics, Foster City, California, USA). Data were analyzed by ANOVA and, as indicated, subject to post-hoc comparisons using two-tailed $t$ test. Values of $P<0.05$ were considered significant.

\section{Results}

To study HC-mediated vascular effects in vivo, we induced HHcy in mice deficient in apoE, in whom hypercholesterolemia and atherosclerosis spontaneously develop (15-16). Mice were fed standard rodent chow (diet $\mathrm{A})$; a diet enriched in methionine, but substantially depleted in folate, vitamins B6, and B12 (diet B); or a diet enriched in methionine and the vitamins folate, B6, and B12 (diet C). After 8 weeks on the diet, mice fed methionine-enriched diet B demonstrated an approximately 19 -fold increase in mean plasma level of HC compared with control mice receiving diet $\mathrm{A}(47.3 \pm 3.2$ vs. $2.5 \pm 0.3 \mu \mathrm{M}$, respectively; $P<$ 0.01 ) (Table 1). Importantly, these levels of HC were pathophysiologically relevant $(2,25)$. For example, in a series of elderly persons studied by the Framingham investigators, levels of plasma $\mathrm{HC}$ ranged from 4.13 to $219.84 \mu \mathrm{M}(2)$. 

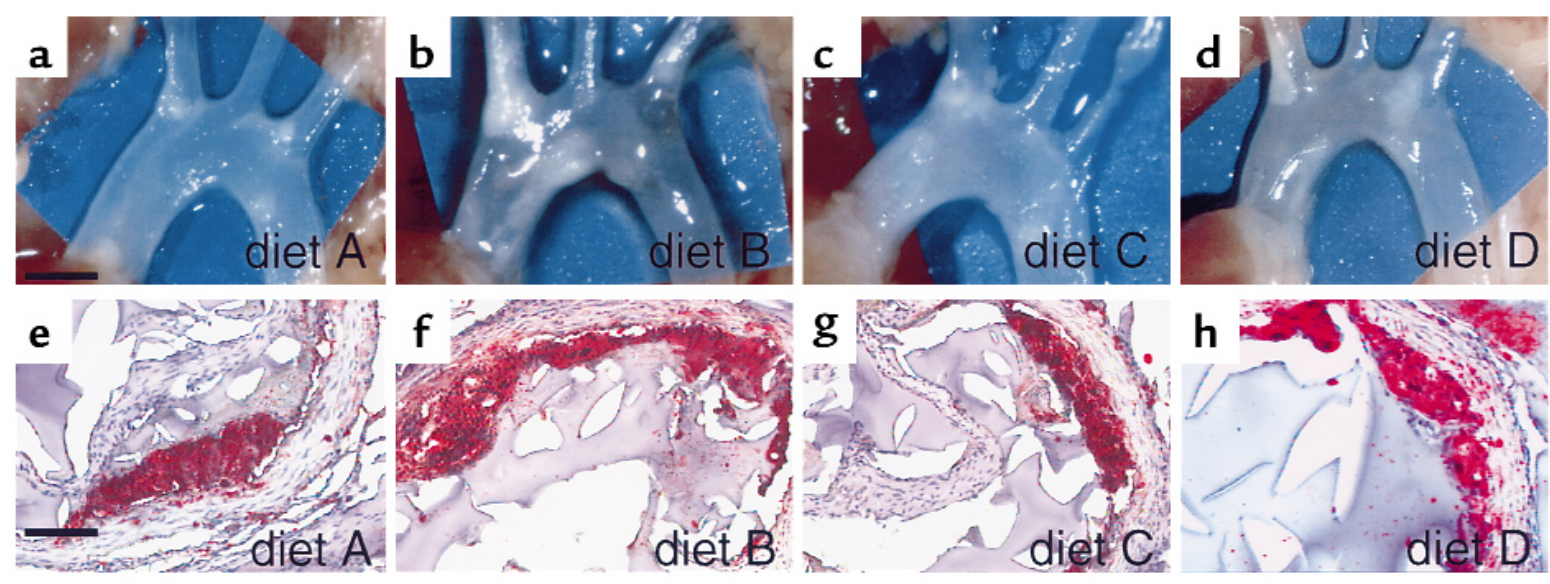

\section{Figure 1}

Induction of $\mathrm{HH} c y$ accelerates atherosclerotic lesion area and complexity. Male apoE-null mice, 4 weeks of age, were fed (a, e) diet A, $n=30$; $(\mathbf{b}, \mathbf{f}) \operatorname{diet} \mathrm{B}, n=30 ;(\mathbf{c}, \mathbf{g}) \operatorname{diet} \mathrm{C}, n=30$, or $(\mathbf{d}, \mathbf{h}) \operatorname{diet} \mathrm{D}, n=10$, for 8 weeks. Mice were sacrificed, and dissection of the aortic tree was performed (scale bar, $2 \mathrm{~mm})(\mathbf{a}-\mathbf{d})$. Serial sections were prepared at the aortic sinus and stained with oil red $\mathrm{O}($ scale bar, $50 \mu \mathrm{m})(\mathbf{e}-\mathbf{h})$. Mean atherosclerotic lesion area (i) and complexity (j) $\pm \mathrm{SD}$ are reported. (i) ${ }^{A} P<$ 0.01 vs. diets $A, C$, and $D .(j){ }^{B} P<0.01$ in numbers of complex lesions vs. those observed in mice receiving diets $A, C$, or $D$. In $\mathbf{i}$ and $\mathbf{j}$, all mice included in the experimental groups as indicated above were analyzed.

Induction of HHcy was associated with an approximately twofold increase in mean atherosclerotic lesion area at the aortic sinus in mice fed diet $\mathrm{B}$ compared with those mice fed control diet $\mathrm{A}$ (Figure $1, \mathrm{~b}$ and $\mathrm{f}$ versus a and e, respectively, and Figure 1i) after 8 weeks. HHcy mice displayed increased numbers of complex atherosclerotic lesions at the aortic sinus, defined as the presence of fibrous caps, cholesterol clefts, or necrosis, compared with controls (Figure 1j). Atherosclerotic lesions in HHcy mice were characterized by increased numbers of macrophages (Mac-3 antigen) (see Figure 5d) and smooth muscle cells (data not shown) compared with mice fed diet A. The effects of HHcy were not due to alteration in lipid or glucose metabolism, as levels of cholesterol, triglyceride, glucose, and glycosylated hemoglobin (a measure of extended glycemic control) were unchanged compared with mice fed control chow (Table 1). Furthermore, separation of plasma lipoprotein components by fast-pressure liquid chromatography (FPLC) revealed no differences in lipid size or profile (data not shown). Importantly, accelerated atherosclerosis observed in mice fed $\operatorname{diet} \mathrm{B}$ was not due to diminished levels of folate, vitamins B6 and $\mathrm{B} 12$, as mice fed diet $\mathrm{D}$ (basal levels of methionine, but decreased levels of folate, vitamins B6/B12) did not display accelerated atherosclerotic lesion area or complexity compared with mice fed $\operatorname{diet} \mathrm{A}$ (Figure 1, $\mathrm{d}$ and $h$ versus a and e, respectively, and Figure $1, i-j)$.

Because these findings suggested that induction of HHcy by dietary enrichment in methionine (diet $B$ ), and not solely dietary depletion of folate and vitamins B6/B12 (diet D), accelerated atherogene-

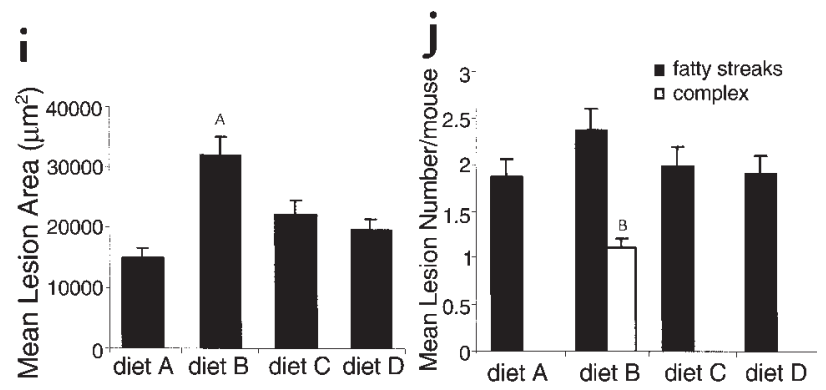

sis, we focused our studies on mice fed diets A and B. To explore the molecular consequences of HHcy in the vasculature, we sought evidence for activation of NF$\kappa \mathrm{B}$, as this transcription factor has been linked to modulation of the proinflammatory response (26-27). Previous studies in vitro demonstrated that incubation of vascular smooth muscle cells (VSMCs) with L-HC $(500 \mu \mathrm{M})$ resulted in enhanced nuclear translocation of NF- $\mathrm{KB}$ (28). Consistent with these observations, exposure of HUVECs to L-HC $(100 \mu \mathrm{M})$ resulted in an approximately 2.4 -fold increase in activation of

Table 1

Induction of HHcy in apoE-null mice: effect on lipids, glucose, and vitamin levels

\begin{tabular}{lcccc}
\hline & Diet A & Diet B & Diet C & Diet D \\
Body weight $(\mathrm{g})$ & $28(3)$ & $27(4)$ & $29(4)$ & $28(3)$ \\
Glucose $(\mathrm{mg} / \mathrm{dl})$ & $115(37)$ & $105(20)$ & $110(2)$ & $112(14)$ \\
Glycosylated & $5.3(0.7)$ & $4.7(0.6)$ & $4.9(0.4)$ & $5.1(0.6)$ \\
hemoglobin $(\%)$ & & & & \\
Cholesterol $(\mathrm{mg} / \mathrm{dl})$ & $315(45)$ & $324(30)$ & $367(52)$ & $310(25)$ \\
Triglyceride $(\mathrm{mg} / \mathrm{dl})$ & $91(31)$ & $64(13)$ & $61(10)$ & $72(19)$ \\
Creatinine $(\mathrm{mg} / \mathrm{dl})$ & $0.3(0.0)$ & $0.3(0.05)$ & $0.25(0.06)$ & $0.3(0.02)$ \\
HC $(\mu \mathrm{M})$ & $2.5(0.3)$ & $47.3(3.2)^{\mathrm{A}}$ & $18.4(2.4)^{\mathrm{B}}$ & $2.4(0.7)$ \\
Folate $(\mathrm{nM})$ & $101(12.3)$ & $8(5.1)^{\mathrm{C}}$ & $53(9.3)^{\mathrm{B}}$ & $10.5(4)^{\mathrm{D}}$ \\
Vitamin B12 $(\mathrm{pM})$ & $5,904(129)$ & $311(91)^{\mathrm{C}}$ & $2,959(135)^{\mathrm{B}}$ & $512(102)^{\mathrm{D}}$ \\
Vitamin B6 (pM) & $270(13)$ & $16.5(1)^{\mathrm{C}}$ & $524(12)^{\mathrm{B}}$ & $11.5(2)^{\mathrm{D}}$
\end{tabular}

Male apo-null mice were fed the indicated diet from the age of 4 weeks through 12 weeks. At sacrifice, plasma was prepared and evaluated for levels of glucose, cholesterol, triglyceride, creatinine, HC, folate, and vitamins B12/B6. Levels of glycosylated hemoglobin were performed on lysates of red blood cells. Data are reported as mean $\pm S D$. ${ }^{A} P<0.01$ vs. $\operatorname{diets} A, C$, and $D .{ }^{B} P<0.05$ vs. $\operatorname{diet} A$ and D. $C P<0.05$ vs. $\operatorname{diet} A$ and $C .{ }^{D} P<0.05$ vs. $\operatorname{diet} A$. 


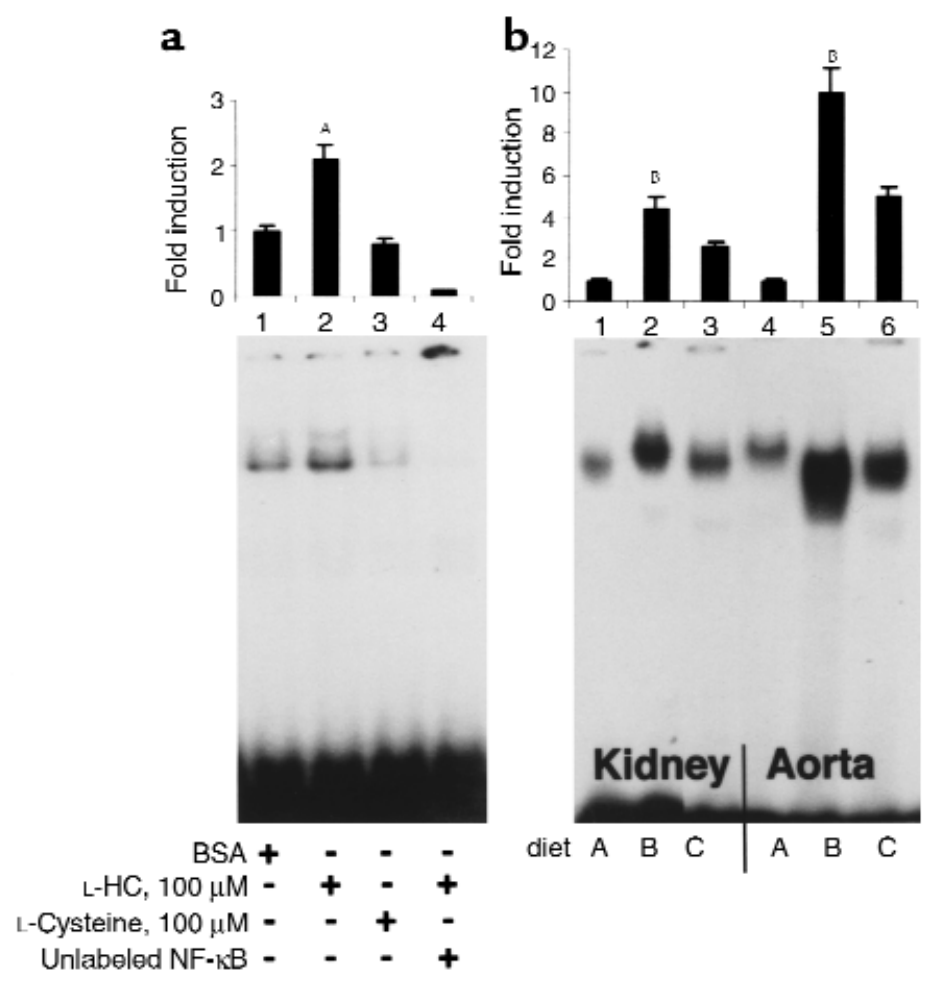

Figure 2

$\mathrm{HH} c y$ enhances activation of NF-KB as seen using EMSA. (a) HUVECs were exposed to BSA, L-HC, or L-cysteine $(100 \mu \mathrm{M})$ for 8 hours. Nuclear extracts were prepared and subjected to EMSA. AP $<0.05$ vs. lanes 1 and 3 . In lane 4, a 100 -fold molar excess of unlabeled NF- $\mathrm{KB}$ was added to indicate specificity for NF-KB. (b) After 8 weeks of diet, apoE-null mice were sacrificed and nuclear extracts prepared from kidney (lanes 1-3) or aorta (lanes 4-6) for EMSA. ${ }^{\mathrm{B}} P<0.01 \mathrm{vs}$. lanes 1 and 3 and 4 and 6 , respectively. EMSA was performed on $n=7 \mathrm{mice} /$ diet; representative experiments are shown. Densitometric analysis was performed; pixels obtained from analysis of EMSA on BSAtreated samples or tissue samples from animals receiving diet A were arbitrarily assigned a relative value of 1 .

NF- $\mathrm{KB}$, compared with ECs treated with BSA or L-cysteine $(100 \mu \mathrm{M})$ (Figure 2a, lanes 2, 1, and 3, respectively). To test these concepts in vivo, we prepared nuclear extracts from aortae and kidney of mice receiving diet $\mathrm{B}$ or A. Compared with mice fed $\operatorname{diet} \mathrm{A}$, animals fed methionine-enriched chow (diet B) displayed an approximately tenfold increase in nuclear translocation of NF- $\mathrm{KB}$ as measured by electrophoretic mobility-shift assay (EMSA) performed on nuclear extracts derived from aorta (Figure 2b, lanes 4 and 5, respectively), and an approximately 4.5 -fold increase in activation of NF- $\mathrm{KB}$ in nuclear extracts prepared from kidney (Figure $2 \mathrm{~b}$, lanes 1 and 2 , respectively).

Since activation of NF- $\mathrm{kB}$ is linked to enhanced expression of genes centrally involved in atherogenesis (29), we explored whether induction of HHcy was associated with increased vascular inflammation. As previous studies suggested that the transcriptional/translational control of expression of VCAM-1, important in the binding of inflammatory mononuclear cells to the vessel wall, is mediated at least in part by activation of NF- $\mathrm{kB}(30-31)$, we assessed levels of this adhesion molecule in vivo. Mice fed methionine-enriched diet B displayed an approximately 3.7 -fold increase in expression of VCAM-1 antigen in aortic tissue compared with mice fed diet A (Figure 3a).

Immunohistochemistry revealed that VCAM1 was expressed in the atherosclerotic lesions of mice fed diet A and B (Figure 3, b and c).

In addition, previous studies suggested that the promoter of the gene encoding RAGE (32), a multiligand signal-transduction receptor of the immunoglobulin superfamily linked to propagation of proinflammatory phenomena (24), possessed functional binding elements for NF-KB (33). In vitro, incubation of HUVEC with L-HC $(100 \mu \mathrm{M})$ resulted in an approximately 2.8-fold increase in expression of RAGE antigen by immunoblotting compared with cells treated with BSA or L-cysteine (Figure 3e). In vivo, aortic tissue retrieved from mice fed diet B displayed an approximately 4.5 -fold increase in expression of RAGE compared with those mice receiving control diet A (Figure 3f). Immunohistochemistry revealed that the expression of RAGE was increased in the atherosclerotic lesions of mice fed diet $B$ versus diet A (Figure 3, h and g, respectively).

EN-RAGEs, members of the S100/calgranulin family of proinflammatory cytokines (34-35) and signal-transducing ligands of RAGE, have been identified in the atherosclerotic plaques of chow-fed apoE-null mice (36). In the presence of HHcy, mice fed $\operatorname{diet} B$ displayed an approximately 3.7 -fold increase in expression of EN-RAGE antigen in the aorta compared with controls, using immunoblotting (Figure 4a). Immunohistochemistry revealed that EN-RAGE was expressed in the atherosclerotic lesions in a manner increased in mice fed diet B compared with diet A (Figure 4, c and b, respectively). Further evidence for HCmediated global cellular activation was demonstrated by the observation that levels of plasma TNF- $\alpha$ were increased 6.1-fold in HHcy mice fed diet B versus those fed control diet A (Figure 4e).

In view of these findings suggesting heightened proinflammatory responses in HHcy vasculature, we assessed levels and activity of tissue-destructive enzymes such as MMPs because previous studies suggested that MMP protein and activity, present in the atherosclerotic plaque, might promote instability and rupture of vascular lesions (37-38). Compared with mice fed diet A, HHcy mice demonstrated an approximately fivefold increase in levels of MMP-9 antigen by immunoblotting (Figure 4f). Importantly, zymography studies revealed increased activity of MMP-9 in aortic tissue obtained from mice with HHcy (diet B) compared with those fed normal chow (Figure 4g). Consistent with these observations, examination of elastic fibers underlying atherosclerotic lesions at the aortic 
sinus stained by van Giessen's method revealed significantly increased elastolysis in mice receiving diet B compared with those mice fed chow (Figure 4h).

In addition to regulation of inflammatory genes, multiple studies suggested that HHcy is associated with altered levels of factors essential for precise control of hemostatic, thrombotic, and fibrinolytic enzymes (39). Even minute differences in levels of such factors likely underlie the observed increased incidence of venous and arterial thromboembolism that occurs in human subjects with HHcy. We tested the concept that exposure of endothelium to HC might alter hemostatic balance, since instability of the atherosclerotic plaque is linked, at least in part, to thrombotic events in the vessel wall (40-41). Since TF is the key trigger of the procoagulant pathway in vivo, its expression in the setting of HHcy was assessed. In vitro, HUVECs exposed to L-HC displayed an approximately 5.2-fold increase in TF antigen by immunoblotting compared with HUVECs treated with L-cysteine $(100 \mu \mathrm{M})$ or BSA (Figure 5a, lanes 2, 3, and 1, respectively). Consistent with these observations, immunoblotting of aortic extracts prepared from HHcy mice fed diet $\mathrm{B}$ revealed an approximately 7.5 -fold increase in levels of procoagulant TF compared with mice fed chow (Figure $5 b$ ).
The expression of TF largely colocalized with that of Mac-3, identifying the macrophage as a prevalent source of TF in the vascular lesions of apoE-null mice with HHcy (Figure 5, g and d, respectively).

Taken together, these findings suggested that induction of HHcy accelerated atherosclerotic lesion formation and complexity and enhanced vascular inflammation, hypercoagulability, and molecular mediators of plaque instability. A central question arising from these observations and epidemiologic investigations in human subjects is whether suppression of HHcy might modulate the course of atherogenesis. To test this, we prepared a diet enriched in methionine, as well as vitamins necessary for the metabolism of $\mathrm{HC}$, folate, and vitamins B6/B12 (diet C). Compared with mice receiving diet $\mathrm{B}$ (19-fold increased $\mathrm{HC}$ ), animals receiving diet C showed only an 7.4-fold increase in HC compared with controls (Table 1 ). The effects of vitamin enrichment in diet $\mathrm{C}$ were evident; levels of folate, vitamins $\mathrm{B} 6$ and B12 were elevated approximately 6.6-fold, 31.2fold, 9.5-fold, respectively, compared with levels observed in mice fed diet B (Table 1). Ingestion of diet $C$ did not result in altered control of glycemia or levels of total cholesterol or triglyceride compared with mice receiving diet $\mathrm{B}$ (Table 1). Furthermore, lipid size/pro-

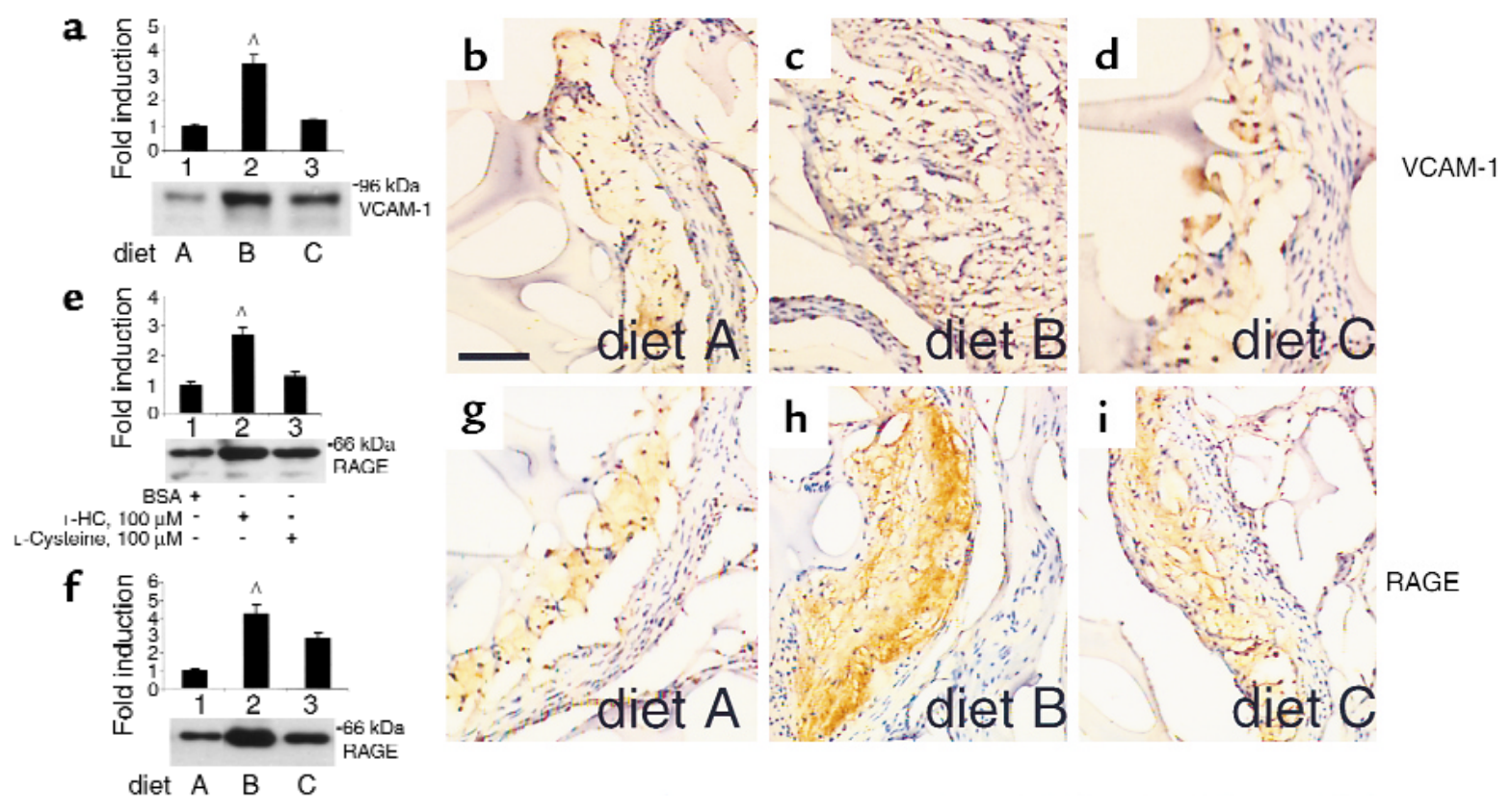

\section{Figure 3}

Induction of HHcy enhances vascular expression of VCAM-1 and RAGE. (a-d) VCAM-1. ApoE-null mice were fed the indicated diet for 8 weeks. Aortae were removed, and lysates were prepared. Lysate protein $(10 \mu \mathrm{g})$ was subjected to immunoblotting with goat anti-VCAM-1 $\operatorname{lgG}(1 \mu \mathrm{g} / \mathrm{ml})$. In a, ${ }^{A} P<0.05$ vs. lanes 1 and 3 . (b-d) Immunohistochemistry was performed using anti-VCAM- 1 lgG ( $\left.8 \mu \mathrm{gg} / \mathrm{ml}\right)$. (e-i) RAGE. In e, HUVECs were exposed to BSA, L-HC, or L-cysteine $(100 \mu \mathrm{M})$ for 8 hours. Lysates were prepared and subjected to immunoblotting with anti-RAGE IgG $(2 \mu \mathrm{g} / \mathrm{ml})$. ${ }^{A} P<0.05 \mathrm{vs}$. lanes 1 and 3 . In $\mathbf{f}$, immunoblotting of aortic lysates was performed using anti-RAGE IgG $(2 \mu \mathrm{g} / \mathrm{ml})$. AP $<0.05$ vs. lanes 1 and 3 . (g-i) Immunohistochemistry was performed using anti-RAGE $\lg G(20 \mu \mathrm{g} / \mathrm{ml})$. In a, e, and $\mathbf{f}$, molecular weight markers are indicated on the right side of each blot. Densitometric analysis was performed; pixel units from aortic tissue derived from mice receiving diet A or HUVECs exposed to BSA were arbitrarily assigned a relative value of 1 . In $\mathbf{a}$ and $\mathbf{f}$, immunoblotting was performed on $n=7$ mice/diet; representative experiments are shown. In $\mathbf{b}-\mathbf{d}$ and $\mathbf{g}-\mathbf{i}$, immunohistochemistry was performed on $n=5$ mice/diet; representative experiments are shown. Scale bar, $50 \mu \mathrm{m}$. 
a
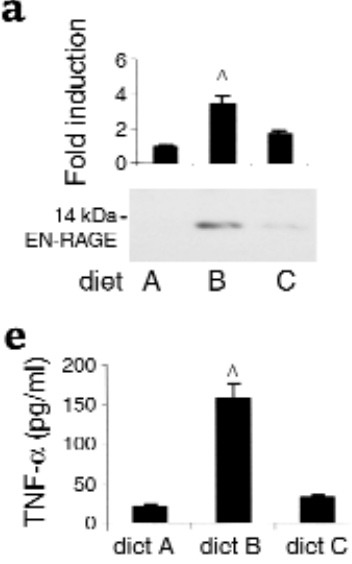
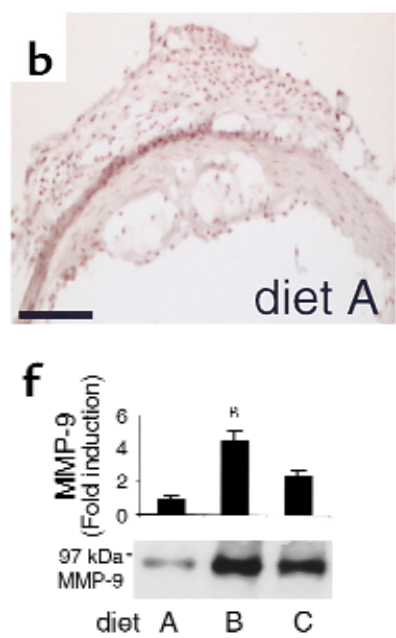

c

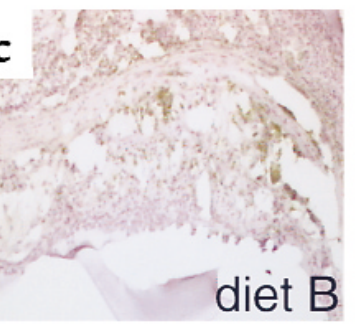

g

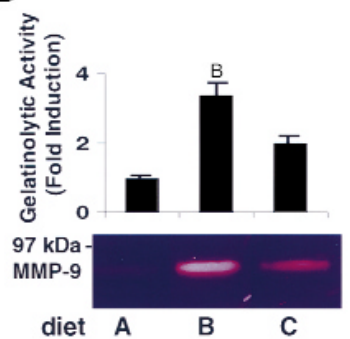

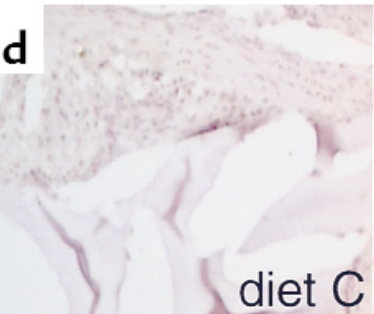

h

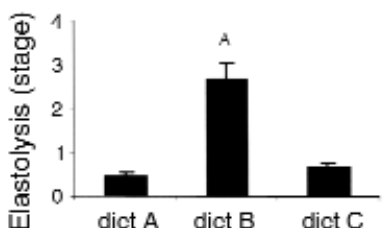

\section{Figure 4}

Induction of HHcy enhances vascular inflammation and expression and activity of MMP-9 and increases elastolysis in aortic tissue from apoE-null mice. ApoE-null mice were fed the indicated diet for 8 weeks. (a-d) EN-RAGE. In a, aortae were removed, and lysates were prepared. Protein $(10 \mu \mathrm{g})$ was subjected to immunoblotting with rabbit anti-EN-RAGE IgG $(2 \mu \mathrm{g} / \mathrm{ml})$. ${ }^{A} P<0.01$ vs. diets $A$ and C. In b-d, immunohistochemistry was performed on atherosclerotic lesions from mice fed diet A, B, or C. Scale bar, $50 \mu \mathrm{m}$. (e) TNF- $\alpha$. Plasma was retrieved from mice, and ELISA was performed for detection of murine TNF- $\alpha$. ${ }^{A} P<0.01$ vs. diets $A$ and $C$. ( $\mathbf{f}$ and $\left.\mathbf{g}\right)$ MMP-9. In $\mathbf{f}$, lysates from aorta were subjected to immunoblotting using anti-MMP-9 $\operatorname{lgG}(1 \mu \mathrm{g} / \mathrm{ml})$. In $\mathbf{g}$, zymography was performed using gelatin-laden gels. In $\mathbf{f}$ and $\mathbf{g},{ }^{B} P<0.05$ vs. diets $A$ and $C$. In $\mathbf{a}, \mathbf{f}$, and $\mathbf{g}$, molecular weight markers are indicated. Densitometric analysis was performed; pixel units from aortic tissue derived from mice receiving diet A were arbitrarily assigned a relative value of 1 . In $\mathbf{a}, \mathbf{e}, \mathbf{f}$, and $\mathbf{g}$, immunoblots or zymograms were performed on $n=7$ mice/diet; representative experiments are shown. In b-d, immunohistochemistry was performed on $n$ $=5 \mathrm{mice} / \mathrm{diet}$; representative experiments are shown. (h). Determination of extent of elastolysis. Lesions were assessed for extent of elastolysis as described above. All lesions in $n=10$ mice/diet were examined. ${ }^{A} P<0.01$ vs. $\operatorname{diets} A$ and $C$.

file determined by FPLC did not differ among mice fed $\operatorname{diet} \mathrm{A}, \mathrm{B}$, or C (data not shown).

We first tested whether reduction of HHcy might modulate atherosclerosis in this model. Mice fed diet C demonstrated only a $40 \%$ increase in lesion area compared with a twofold increase observed in mice fed diet $\mathrm{B}$ (Figure 1, $\mathrm{c}$ and $\mathrm{g}$ and Figure 1, $\mathrm{b}$ and $\mathrm{f}$, respectively; and Figure 1i). Furthermore, lesions in apoE-null mice fed diet $\mathrm{C}$ were limited to fatty streaks; no complex lesions were noted (Figure 1j).

These observations suggested that suppression of accelerated atherosclerosis in mice fed $\operatorname{diet} \mathrm{C}$ was associated with diminished vascular activation. To test this concept, we explored the effects of reduced HC levels on putative pathogenic mechanisms underlying the effects of HHcy. Consistent with the premise that HHcy mediated enhanced vascular inflammation, nuclear extracts prepared from mice fed diet $\mathrm{C}$ displayed significantly decreased nuclear translocation of NF- $\mathrm{KB}$ in the aorta and kidney compared with mice fed diet B (Figure 2b, lanes 6 and 5 and lanes 3 and 2, respectively).

In parallel with diminished activation of NF- $\mathrm{\kappa B}$ in vascular tissue from mice fed diet $\mathrm{C}$, immunoblotting studies revealed that the expression of VCAM-1, RAGE, and EN-RAGE antigens in aortic tissue was significantly diminished compared with that observed in mice fed diet B (Figure 3, a and f, and Figure 4a). In addition, lev- els of plasma TNF- $\alpha$ in mice fed diet $C$ were reduced nearly to levels observed in mice fed diet A (Figure 4e). Similarly, expression/activity of MMP-9 were reduced in mice fed diet $\mathrm{C}$ versus diet $\mathrm{B}$ (Figure 4, $\mathrm{f}$ and $\mathrm{g}$ ). Consistent with this observation, examination of elastic fibers underlying atherosclerotic plaques in lesions from mice fed diet $C$ revealed decreased elastolysis compared with mice with HHcy fed diet B (Figure 4h).

Lastly, since plaque instability and rupture markedly alter the clinical course of atherosclerosis, we assessed levels of likely contributory molecules in this model. Compared with mice fed diet B, those fed diet C demonstrated an approximately twofold decrease in levels of TF in the aorta (Figure 5b).

\section{Discussion}

Our findings suggest that the biochemical and molecular consequences of HHcy appear far-reaching, from enhanced activation of NF- $\mathrm{KB}$ in the vasculature to modulation of expression of a range of genes that may disrupt homeostatic anti-inflammatory and antithrombotic mechanisms. The present model in HHcy apoE-null mice suggests that induction of HHcy induces enhanced expression/activity of key participants in vascular inflammation, atherogenesis, and vulnerability of the established atherosclerotic plaque. These data thus support the concept of a "two-hit 
model," as HHcy modulates increased risk for vascular lesion development and progression, likely by exacerbating risk associated with traditional factors such as hyperlipidemia. In this context, the observed increase in oxidation of LDL in the presence of increased HC supports this view (8).

Previous investigation suggested that expression of a range of genes might be modulated by excess $\mathrm{HC}$. For example, in cultured HUVECs exposed to HC, cDNA microarray analyses indicated increased stress within the endoplasmic reticulum (ER), as levels of a number of ER stress proteins, such as GADD153, were upregulated (42-43). Furthermore, along with other genes whose expression was enhanced upon exposure to HC, the expression of genes that regulate antioxidant defenses in the cell, such as natural kill-enhancing factor $\beta$, glutathione peroxidase, and superoxide dismutase, was diminished (44). Although yet to be fully confirmed in vivo, these findings nevertheless support the hypothesis that chronic exposure to elevated levels of $\mathrm{HC}$ may prompt vascular perturbation.

In addition to these HC-modulated genes identified by microarray analysis, the present findings have demonstrated increased expression of RAGE and its key ligand in inflammatory settings, EN-RAGEs, in HHcy mice. These observations strongly suggest that HHcy induces chronic cellular activation and dysfunction, as our previous studies identified that expression of RAGE is enhanced and sustained in settings in which its ligands form and accumulate. For example, in diabetes, the striking upregulation and colocalization of RAGE, AGEs, and EN-RAGEs is associated with a heightened state of activation in such cells as the endothelium, VSMCs, monocytes, and neurons (32). Sustained expression of RAGE augurs a range of complications, including vascular hyperpermeability, accelerated atherosclerosis, and exaggerated responses to oral infection $(19,32,45)$. We thus speculate that increased expression of RAGE and EN-RAGEs in the vasculature of HHcy mice provides one mechanism for chronic cellular dysfunction.

Recently, the intriguing viewpoint was raised that elevated levels of plasma HC may represent, at least in part, the effect, and not solely the cause, of vascular dysfunction (46). Based on the concept that $\mathrm{HC}$ may be released from damaged tissues, since repair of DNA, RNA, and protein involves methylation and increased generation of S-adenosylhomocysteine (SAH) and $\mathrm{HC}$ within the cell, this view highlights the possibility that a spiraling cascade of chronic vascular inflammation, injury, and repair may be set in motion by elevated levels of plasma $\mathrm{HC}$ and other vascular risk factors, such as hypercholesterolemia. Although this concept has yet to be aggressively tested in vitro or in vivo, the model presented here provides a framework for the dissection of the time- and dose-dependent effects of elevated $\mathrm{HC}$ in vivo.

Irrespective of the etiology of HHcy, multiple studies have underscored a critical link between plasma $\mathrm{HC}$ and the levels of vitamins required for its metabolism (17-18). Although plasma folate is likely the most
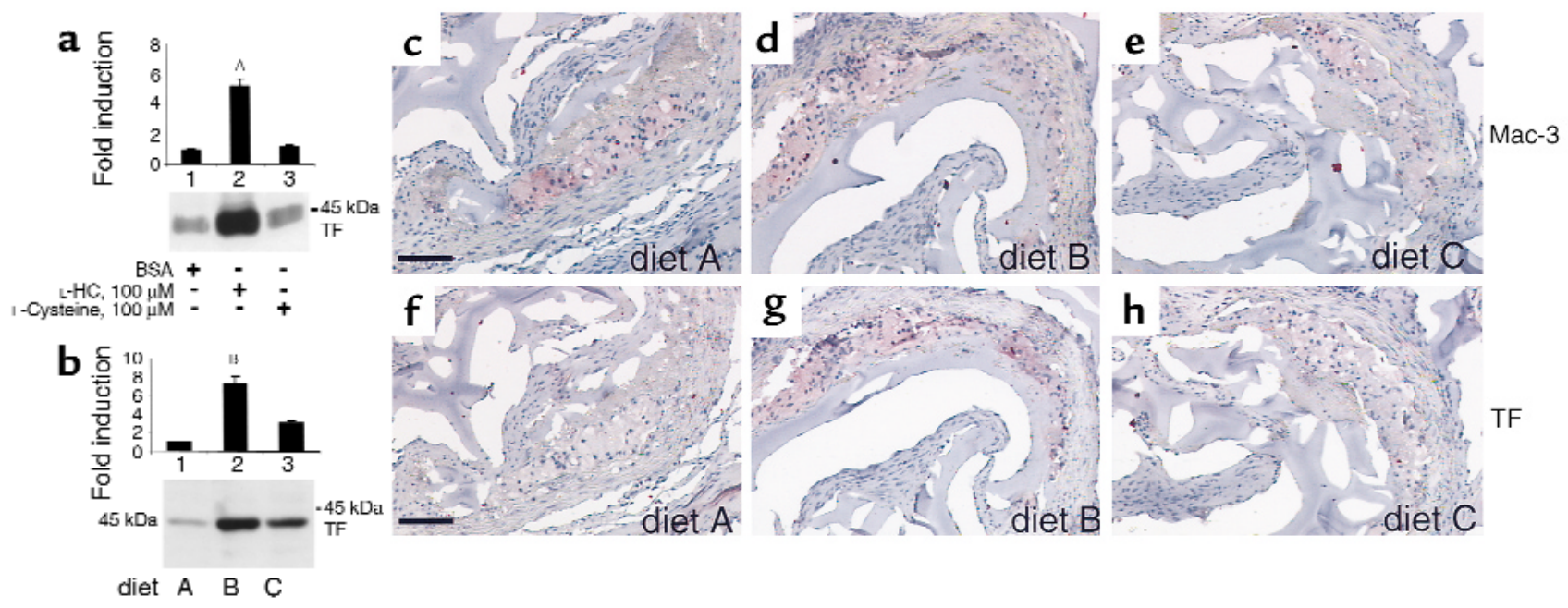

\section{Figure 5}

Induction of HHcy enhances expression of TF. (a) HUVECs were exposed to the indicated concentration of BSA, L-HC, or L-cysteine for 8 hours. Cells were harvested and prepared for immunoblotting using goat anti-rat TF IgG $(0.5 \mu \mathrm{g} / \mathrm{ml})$. ${ }^{A} P<0.05$ vs. lanes 1 and 3 . (b-h) ApoE-null mice were sacrificed after 8 weeks of the indicated diet, aortae were removed, and lysates were prepared. Protein $(10 \mu \mathrm{g})$ was subjected to SDS-PAGE and transfer to nitrocellulose. Immunoblotting was performed with goat anti-rat TF lgG ( $1 \mu \mathrm{g} / \mathrm{ml})$. Molecular weight markers are indicated. Densitometric analysis was performed; pixel units from aortic tissue derived from mice receiving diet A or BSA-treated HUVECs were arbitrarily assigned a relative value of $1 .{ }^{B} P<0.01$ vs. diets $A$ and $C$. In $\mathbf{b}$, immunoblotting on lysates from $n=10$ mice per diet was performed; representative experiments are shown. In $\mathbf{c}-\mathbf{h}$, apoE-null mice were fed $\operatorname{diet} A(\mathbf{c}, \mathbf{f})$, diet B $(\mathbf{d}, \mathbf{g})$, or $\operatorname{diet} \mathrm{C}(\mathbf{e}, \mathbf{h})$, for 8 weeks. Upon sacrifice, serial sections at the aortic sinus were prepared and stained with anti-Mac-3 IgG $(\mathbf{c}-\mathbf{e})(10 \mu \mathrm{g} / \mathrm{ml})$ or goat anti-rat TF $\mathbf{l g G}(\mathbf{f}-\mathbf{h})(10 \mu \mathrm{g} / \mathrm{ml})$. In $\mathbf{c}-\mathbf{h}$, immunohistochemistry was performed on $n=5$ mice/diet; representative experiments are shown. Scale bar, $50 \mu \mathrm{m}$. 
important determinant of plasma HC level, it is probably not the sole factor (17). In a previous study, Ambrosi and colleagues reported that induction of HHcy in pigs enhanced development of arterial lesions and elastolysis (47). In that model, ingestion of methionineenriched diet and folic acid ( $5 \mathrm{mg} /$ day), the latter begun 1 month after commencement of the diet, did not modulate the course of vascular dysfunction. However, it is not clear that levels of $\mathrm{HC}$ were sufficiently reduced nor that plasma levels of the vitamin were significantly enhanced in those studies. Lack of dietary enrichment with vitamins B6/B12 might too have contributed to the apparent lack of efficacy of folate therapy. In support of the concept that vitamin supplementation may modulate HHcy and its consequences in the vasculature, a recent report suggested that treatment of human subjects with HHcy with folate and vitamins B6/B12 resulted in attenuation of thrombin generation, both in the peripheral blood and at sites of hemostatic plug formation (48). Supplementation with folate and vitamin B6 also resulted in diminished levels of putative markers of endothelial perturbation, soluble thrombomodulin, and von Willebrand factor (49). Recently, lowering of plasma $\mathrm{HC}$ by dietary supplementation with folate and vitamin B6 for 2 years in human subjects resulted in decreased incidence of abnormal exercise electrocardiography studies (50). Administration of folate and vitamin B12 for 9 weeks to men with coronary heart disease and HHcy was shown to improve vascular endothelial function, as assessed by brachial artery flow-mediated dilatation (51).

Lastly, recent studies in a murine model of genetically mediated HHcy employing mice with heterozygous deletion of the gene encoding cystathionine- $\beta$ synthase (CBS) (52) revealed that HHcy was associated with both enhanced oxidant stress in the vasculature, as well as endothelial dysfunction (53). The present findings complement and extend those observations and point to elevated plasma $\mathrm{HC}$ as a key culprit in the development of vascular dysfunction and the steps leading to accelerated atherosclerosis. In conclusion, we demonstrated that induction of HHcy in apoE-null mice by dietary modulation of methionine, folate, and vitamins B6/B12 accelerated vascular inflammation, hypercoagulability, and atherosclerosis. The observation that dietary enrichment in folate and vitamins B6/B12 suppressed plasma levels of HC in parallel with markers of vascular dysfunction, strongly suggests that dietary supplementation with these vitamins might have long-term beneficial effects. Studies are underway to delineate which one(s) of these vitamins provides protective effects in the vasculature in this model, and whether introduction of these vitamins after a period of HHcy may modulate the course of atherogenesis and/or induce lesion regression. This model may serve as a springboard for the development of public health policy in this area, especially as we await the results of long-term clinical trials of vitamin supplementation in human subjects.

\section{Acknowledgments}

This work was supported, in part, by the Surgical Research Fund of the College of Physicians \& Surgeons, Columbia University, and by grants from the United States Public Health Service, Juvenile Diabetes Foundation International, and the American Heart Association, New York affiliate. M.A. Hofmann is a recipient of postdoctoral fellowship award from the Juvenile Diabetes Foundation. A.M. Schmidt is a recipient of a Burroughs Wellcome Fund Clinical Scientist Award in Translational Research.

1. Eikelboom, J.W., Lonn, E., Genest, J., Jr., Hankey, G., and Yusuf, S. 1999. Homocyst(e)ine and cardiovascular disease: a critical review of the epidemiologic evidence. Ann. Intern. Med. 131:363-375.

2. Bostom, A.G., et al. 1999. Nonfasting plasma total homocysteine levels and stroke incidence in elderly persons: the Framingham study. Ann. Intern. Med. 131:352-355.

3. Harker, L.A., Slichter, S.J., Scott, C.R., and Ross, R. 1974. Homocystinemia. Vascular injury and arterial thrombosis. N. Engl. J. Med. 291:537-543.

4. Loscalzo, J. 1996. The oxidant stress of hyperhomocyst(e)inemia. J. Clin. Invest. 98:5-7.

5. Stamler, J.S., et al. 1993. Adverse effects of homocysteine are modulated by endothelium-derived relaxing factor and related oxides of nitrogen. J. Clin. Invest. 91:308-318.

6. Kanani, P.M., et al. 1999. Role of oxidant stress in endothelial dysfunction produced by experimental hyperhomocyst(e)inemia in humans. Circulation. 100:1161-1168.

7. Lubec, B., et al. 1996. Homocysteine increases cyclin-dependent kinase in aortic rat tissue. Circulation. 94:2620-2625.

8. Hirano, K., et al. 1994. Homocysteine induces iron-catalyzed lipid peroxidation of low density lipoprotein that is prevented by alpha-tocopherol. Free Radic. Res. 21:267-276.

9. Durand, P., Lussier-Cacan, S., and Blache, D. 1997. Acute methionine load-induced hyperhomocysteinemia enhances platelet aggregation, thromboxane biosynthesis, and macrophage-derived tissue factor. FASEB J. 11:1157-1168.

10. Rodgers, G.M., and Kane, W.H. 1986. Activation of endogenous factor $V$ by a homocysteine-induced vascular endothelial cell activator. J. Clin. Invest. 77:1909-1916.

11. Rodgers, G.M., and Conn, M.T. 1990. Homocysteine, an atherogenic stimulus, reduces protein $\mathrm{C}$ activation by arterial and venous endothelial cells. Blood. 75:895-901.

12. Lentz, S.R., and Sadler, J.E. 1991. Inhibition of thrombomodulin surface expression and protein $\mathrm{C}$ activation by the thrombogenic agent homocysteine. J. Clin. Invest. 88:1906-1914.

13. Hajjar, K.A., et al. 1998. Tissue plasminogen activator binding to the annexin II tail domain. Direct modulation by homocysteine. J. Biol. Chem. 273:9987-9993.

14. Hajjar, K.A., and Jacovina, A.T. 1998. Modulation of annexin II by homocysteine: implications for atherothrombosis. J. Investig. Med. 46:364-369.

15. Zhang, S.H., Reddick, R.L., Piedrahita, J.A., and Maeda, N. 1992. Spontaneous hypercholesterolemia and arterial lesions in mice lacking apolipoprotein E. Science. 258:468-471.

16. Plump, A.S., et al. 1992. Severe hypercholesterolemia and atherosclerosis in apolipoprotein $\mathrm{E}$ deficient mice created by homologous recombination in ES cells. Cell. 71:343-353.

17. Boushey, C.J., Beresford, S.A., Omenn, G.S., and Motulsky, A.G. 1995. A quantitative assessment of plasma homocysteine as a risk factor for vascular disease. Probable benefits of increasing folic acid intakes. JAMA. 274:1049-1057.

18. Verhoef, P., et al. 1996. Homocysteine metabolism and risk of myocardial infarction: relation with vitamins B6, B12 and folate. Am. J. Epidemiol. 149:845-859.

19. Park, L., et al. 1998. Suppression of accelerated diabetic atherosclerosis by the soluble receptor for advanced glycation endproducts. Nat. Med. 4:1025-1031.

20. Prescott, M.F., et al. 1999. Effect of matrix metalloproteinase inhibition on progression of atherosclerosis and aneurysm in LDL receptor deficient mice overexpressing MMP, MMP-12, and MMP-13 and on restenosis in rats after balloon injury. Ann. NY Acad. Sci. 878:179-190.

21. Carmeliet, P., et al. 1997. Urokinase-generated plasmin activates matrix metalloproteinases during aneurysm formation. Nat. Genet. 17:439-444.

22. Vester, B., and Rasmussen, K. 1991. High performance liquid chromatography method for rapid and accurate determination of homocysteine in plasma and serum. Eur. J. Clin. Chem. Clin. Biochem. 29:549-554. 
23. Schreck, R., Grassmann, R., Fleckenstein, B., and Baeuerle, P.A. 1992. Antioxidants selectively suppress activation of NF-kappaB by human $\mathrm{T}$ cell leukemia virus type 1 Tax protein. J. Virol. 66:6288-6293.

24. Hofmann, M.A., et al. 1999. RAGE mediates a novel proinflammatory axis: a central cell surface receptor for S100/calgranulin polypeptides. Cell. 97:889-901.

25. Dierkes, J., et al. 1999. Response of hyperhomocysteinemia to folic acid supplementation in patients with end-stage renal disease. Clin. Nephrol. 51:108-115.

26. Schreck, R., Albermann, K., and Baeuerle, P.A. 1992. Nuclear factor kappa B: an oxidative stress-responsive transcription factor of eukaryotic cells. Free Radic. Res. Commun. 17:221-237.

27. Kretz-Remy, C., Mehlen, P., Mirault, M.E., and Arrigo, A.P. 1996. Inhibition of I kappa B alpha phosphorylation and degradation and subsequent NF-kB activation by glutathione peroxidase overexpression. J. Cell Biol. 133:1083-1093.

28. Welch, G.N., et al. 1998. Homocysteine-induced nitric oxide production in vascular smooth-muscle cells by NF-KB-dependent transcriptional activation of Nos2. Proc. Assoc. Am. Physicians. 110:22-31.

29. Collins, T. 1993. Endothelial nuclear factor kappa B and the initiation of the atherosclerotic lesion. Lab. Invest. 68:499-508.

30. Marui, N., et al. 1993. VCAM-1 gene transcription and expression are regulated through an oxidant-sensitive mechanism in human vascular endothelial cells. J. Clin. Invest. 92:1866-1874.

31. Neish, A., Williams, A., Palmer, H., Whitley, M., and Collins, T., 1992. Functional analysis of the human VCAM-1 promoter. J. Exp. Med. 176:1583-1593

32. Schmidt, A.M., Yan, S.D., Wautier, J.L., and Stern, D. 1999. Activation of receptor for advanced glycation endproducts: a mechanism for chronic vascular dysfunction in diabetic vasculopathy and atherosclerosis. Circ. Res. 84:489-497.

33. Li, J., and Schmidt, A.M. 1997. Characterization and functional analysis of the promoter of RAGE, the receptor for advanced glycation endprod ucts. J. Biol. Chem. 272:16498-16506.

34. Schafer, B.W., and Heinzmann, C.W. 1996. The S100 family of EF-hand calcium-binding proteins: functions and pathology. Trends Biochem. Sci. 21:134-140.

35. Zimmer, D.B., Cornwall, E.H., Landar, A., and Song, W. 1995. The S100 protein family: history, function, and expression. Brain Res. Bull. 37:417-429.

36. Bobryshev, Y.V., Babaev, V.R., Iwasa, S., Lord, R.S., and Watanabe, T. 1999. Atherosclerotic lesions of apolipoprotein $\mathrm{E}$ deficient mice contain cells expressing S100 protein. Atherosclerosis. 143:451-454.

37. Aikawa, M., et al. 1998. Lipid lowering by diet reduces matrix metalloproteinase activity and increases collagen content of rabbit atheroma: a potential mechanism of lesion stabilization. Circulation. 23:2433-2444.

38. Mach, F., Schonbeck, U., Bonnefoy, J.Y., Pober, J.S., and Libby, P. 1997. Activation of monocyte/macrophage functions related to acute atheroma complication by ligation of CD40: induction of collagenase, stromelysin, and tissue factor. Circulation. 15:396-399.

39. Eichinger, S., et al. 1998. Hyperhomocysteinemia is a risk factor of recurrent venous thromboembolism. Thromb. Haemost. 80:566-569.

40. Tremoli, E., Camera, M., Toschi, V., and Colli, S. 1999. Tissue factor in atherosclerosis. Atherosclerosis. 144:272-283.

41. Taubman, M.B., et al. 1997. Tissue factor in the pathogenesis of atherosclerosis. Thromb. Haemost. 78:200-204.

42. Kokame, K., Kato, H., and Miyata, T. 1996. Homocysteine-respondent genes in vascular endothelial cells identified by differential display analysis. GFP78/BiP and novel genes. J. Biol. Chem. 271:29659-29665.

43. Outinen, P.A., et al. 1999. Homocysteine-induced endoplasmic reticulum stress and growth arrest leads to specific changes in gene expression in human vascular endothelial cells. Blood. 94:959-967.

44. Chacko, G., Ling, Q., and Hajjar, K.A. 1998. Induction of acute translational response genes by homocysteine. Elongation factors-1alpha, -beta, and -delta. J. Biol. Chem. 273:19840-19846.

45. Wautier, J.L., et al. 1996. Receptor-mediated endothelial cell dysfunction in diabetic vasculopathy. Soluble receptor for advanced glycation endproducts blocks hyperpermeability in diabetic rats. J. Clin. Invest. 97:238-243.

46. Dudman, N.P. 1999. An alternative view of homocysteine. Lancet. 354:2072-2074.

47. Ambrosi, P., et al. 1999. Effects of folate supplementation in hyperhomocysteinemic pigs. J. Am. Coll. Cardiol. 34:274-279.

48. Undas, A., Domagala, T.B., Jankowski, M., and Szczeklik, A. 1999. Treatment of hyperhomocysteinemia with folic acid and vitamins B6 and B12 attenuates thrombin generation. Thromb. Res. 96:281-288.

49. Constans, J., et al. 1999. Three months supplementation of hyperhomocysteinemic patients with folic acid and vitamin B6 improves biological markers of endothelial dysfunction. Br. J. Haematol. 107:776-778.

50. Vermeulen, E.G., et al. 2000. Effect of homocysteine-lowering treatment with folic acid plus vitamin B6 on progression of subclinical atherosclerosis: a randomized, placebo-controlled trial. Lancet. 355:517-522.

51. Chambers, J.C., et al. 2000. Improved vascular endothelial function after oral B vitamins: an effect mediated through reduced concentrations of free plasma homocysteine. Circulation. 102:2479-2483.

52 . Watanabe, M., et al. 1995 . Mice deficient in cystathione- $\beta$-synthase: animal models for mild and severe homocyst(e)inemia. Proc. Natl. Acad. Sci. USA. 92:1585-1589.

53. Eberhardt, R.T., et al. 2000. Endothelial dysfunction in a murine model of mild hyperhomocyst(e)inemia. J. Clin. Invest. 106:483-491. 